\title{
Cryptogenic Stroke and Significance of the Patent Foramen Ovale: A Case Series
}

\author{
Pour-Ghaz I*, Jackson $\mathbf{C}^{1}$, Bhole $\mathbf{R}^{2}$, Krishnan $\mathbf{R}^{2}$, Pierce WF${ }^{3}$ and Seth $\mathrm{D}^{4}$ \\ ${ }^{1}$ Department of Internal Medicine, University of Tennessee Health Science Center, Memphis, TN, USA \\ ${ }^{2}$ Department of Neurology, University of Tennessee Health Science Center, Memphis, TN, USA \\ ${ }^{3}$ Department of Medicine, University of Tennessee College of Medicine, Memphis, TN, USA \\ ${ }^{4}$ Department of Medicine, University of Tennessee at Baptist Memorial Hospital, Memphis, TN, USA
}

\begin{abstract}
Stroke is the second leading cause of death globally and can lead to significant adverse outcomes in patients following the acute illness. Due to this high morbidity and mortality, adequate interventions can play a significant role in health outcomes. Patent foramen ovale is one of the major proposed causes of cryptogenic strokes and can be present in up to $25 \%$ of general population. In cryptogenic strokes, the relation of this structural heart defect is inversely proportional to age of patient, whereby, it plays a more significant role in the etiology of the cryptogenic strokes in younger patients. In this paper we present three cases of cryptogenic strokes in patients with patent foramen ovale and discuss the impact of such finding and treatment for such cases. We demonstrate that in the younger age spectrum, patent foramen ovale plays a more significant role and treatment can prevent future stroke episodes.
\end{abstract}

Keywords: Patent foramen ovale; Cryptogenic stroke; Transient ischemic attack; PFO; PFO closure

\section{Introduction}

Stroke is the second leading cause of death globally and one of the important causes of morbidity and mortality in the United States [1]. Cryptogenic strokes consist of a subset of strokes where no probable cause is identified despite adequate diagnostic evaluations. Cryptogenic strokes can be subclassified into 'highly cryptogenic' and those of 'possibly determined origin' [2]. Cryptogenic strokes account for approximately $25 \%$ of strokes, which is a significant number of cases without an identifiable cause, thus, treating patients for secondary prevention is essential. Cryptogenic stroke is a diagnosis of exclusion and can only be diagnosed after workup for other causes such as large artery atherosclerosis, small artery disease, cardioembolic and structural cardiac abnormalities, have been ruled out [3]. Hence, the term cryptogenic stroke is used to describe the absence of any other known cause of stroke except patent foramen ovale (PFO). Moreover, it has been established that PFO prevalence is significantly higher in patients who have cryptogenic strokes [4]. As a result, when a cryptogenic stroke is an underlying cause for stroke, closure of the PFO would be the next step. However, there have been multiple randomized trials in the earlier years which failed to show the benefit of PFO closure. More recently, trials have shown that long-term outcome of PFO closure reduces the recurrence of strokes. CLOSE trial (Patent Foramen Ovale Closure or Anticoagulants versus Antiplatelet Therapy to Prevent Stroke Recurrence; NCT00562289) showed that percutaneous PFO closure was associated with significantly fewer recurrent strokes in patients with cryptogenic stroke which was also the result of the RESPECT trial (Randomized Evaluation of Recurrent Stroke Comparing PFO Closure to Established Current Standard of Care Treatment; NCT00465270). RESPECT trial showed that PFO closure significantly reduced the incidence of recurrent stroke compared with medical therapy $[5,6]$. It is important to note that choosing which patients get PFO closure through risk stratification is vital for such patients after adequate work up is done to exclude other causes for stroke. Here, we will present three cases that demonstrate why early PFO closure, especially in younger patients, can be beneficial.

\section{Case Reports}

\section{Case 1}

A 30-year-old African American male with past medical history of insulin dependent diabetes mellitus, hypertension, sleep apnea and a recent myocardial infarction 7 -months prior presents with acute onset dysarthria and angioedema secondary to lisinopril use. His angioedema was treated appropriately, and stroke team was consulted for concerns due to dysarthria. The patient also commented that he was having intermittent right sided upper extremity paresthesia's. A non-contrast computer tomography (CT) of head was obtained, which demonstrated a subacute right parietal cortical hypodensity. Since his previous myocardial infarction, the patient had been complaint with his aspirin and Plavix. He noted that approximately 1 month ago, he suffered a severe headache, but did not remember which side it was on, what precipitated this headache or how long it lasted. Review of systems was negative except for the angioedema and dysarthria. His labs on presentation were LDL 123, HbA1c 7.2, hemoglobin and hematocrit of 20.9 and 65.7 with P2Y1288. A magnetic resonance imaging (MRI) was performed, which showed no evidence of diffusion restriction to suggest recent infarction. Encephalomalacia was demonstrated involving the right parietal lobe secondary to sequela from remote infarction. Computer tomography angiography (CTA) was negative for any pathology including any significant stenosis. Transthoracic echocardiogram (TTE) showed a left ventricle (LV) with normal size and normal systolic function. LV ejection fraction was 55-60\% with normal regional wall motion, normal diastolic function, and no LV thrombus. There was no evidence of atrial septal defect, ventricular septal defect or PFO. At this time the

*Corresponding author: Pour-Ghaz I, Department of Internal Medicine, University of Tennessee Health Science Center, Memphis, TN, USA, Tel: (901) 671-5125; E-mail: ipourgha@uthsc.edu

Received August 31, 2018; Accepted September 06, 2018; Published September 11, 2018

Citation: Pour-Ghaz I, Jackson C, Bhole R, Krishnan R, Pierce WF, et al. (2018) Cryptogenic Stroke and Significance of the Patent Foramen Ovale: A Case Series. J Clin Case Rep 8: 1167. doi: 10.4172/2165-7920.10001167

Copyright: @ 2018 Pour-Ghaz I, et al. This is an open-access article distributed under the terms of the Creative Commons Attribution License, which permits unrestricted use, distribution, and reproduction in any medium, provided the origina author and source are credited. 
recommendations were cardiac event monitor and outpatient follow up since symptoms were consistent with a transient ischemic attack (TIA) versus dysarthria secondary to the angioedema, both of which required no intervention.

Approximate 36 hours after stroke team visit, the patient was found to have a right-sided facial droop and right-sided arm weakness with last known well reportedly overnight. NIHSS was 4 (right facial droop, dysarthria, right upper extremity drift) and stroke team was reconsulted. MRI showed interval development of diffusion restriction involving the left parietal lobe and there was associated signal hypointensity on the apparent diffusion coefficient map most characteristic of recent infarction. This acute left parietal small ischemic stroke was out of window for tissue plasminogen activator administration. Transesophageal echocardiogram (TEE) with saline contrast injection was performed at rest with good effort Valsalva which demonstrated at least 20 early appearing left heart bubbles, consistent with present of a large size PFO. Magnetic resonance angiography showed no evidence for pelvic or common femoral venous thrombosis. The stroke was attributed to a large inter-atrial communication defect with etiology being paradoxical embolus through defect. Patient was considered for transcatheter PFO closure, however, patient opted for outpatient closure of defect. He was sent home with close follow up and event monitor together with atorvastatin and dual antiplatelet therapy.

\section{Case 2}

A 66-year-old Caucasian male with past medical history significant for hypertension and hyperlipidemia presented with altered mental status. Symptoms at presentation included slurred speech. He was found walking around his yard and his neighbor noted his slurred speech. His last know well state was the day before in the evening hours. Upon arrival, the blood pressure was found to be at $150 / 70$ and his laboratory work up including complete blood count, complete metabolic profile and urinalysis were normal. Urine drug screen was positive for cannabinoids and serum alcohol level was below 3. CT of the head showed age related atrophy and mild small vessel ischemic changes. Questionable loss of gray-white junction and edema was found in the left frontoparietal region. On physical exam, the patient was confused but had normal movements and strength. He denied use of any tobacco products. TTE showed a normal left ventricle size, thickness, and function. The ejection fraction was estimated at $60 \%$ with normal right ventricular structure and function. There was a small PFO with predominant left-to-right shunting visualized. MRI showed an acute left middle cerebral artery territory infarction without midline shift or hemorrhagic conversion. CTA showed the right internal carotid artery with estimated stenosis of $30-50 \%$ and the left internal carotid artery with stenosis estimated at $30-50 \%$. TEE showed the PFO previously demonstrated and an ejection fraction of approximately $60 \%$. Ultrasound of lower extremities failed to show deep vein thrombosis. Patient continued receiving neuro checks during the length of the stay and was started on antiplatelet therapy and atorvastatin with an outpatient follow up and discharged with an event recorder and possible percutaneous closure of PFO after conduction abnormalities were ruled out.

\section{Case 3}

An 18-year-old Caucasian female with past medical history significant for Raynaud's phenomena and vascular headaches, which have never been associated with any neurologic abnormalities, presented after an episode of not being able to articulate and not being able to feel her right arm and the right side of her face. During this episode, the patient had a unilateral frontal headache and a brief episode of lightheadedness. The episode lasted 2 minutes after which she returned to baseline. Patient was on oral contraceptive pills until this episode. On examination, patient did not have any significant physical findings. TTE showed a normal ejection fraction of $65 \%$ with normal diastolic function and the presence of a PFO. CTA brain with and without contrast did not show any stenosis, dissection, or aneurysm. MRI of the brain with and without contrast showed no acute intracranial findings. Patient underwent placement of an Amplatzer closure device and was followed up with repeat TEE which showed an ejection fraction greater than 55\% and no evidence of spontaneous echo contrast in the left atrium. Repeat echocardiography showed that the Amplatzer device was in good position. On follow-up visits, the patient did not have any new episodes of a cryptogenic stroke. Patient had the last echocardiography done in February 2018, which showed that the ejection fraction was greater than $65 \%$ and that the atrial septal occluder device was in a stable position with no residual shunting. There was no evidence of device migration. The remaining valves were grossly normal. Since the placement of the closing device, the patient has been doing well without any residual neurologic defects or repeated episodes of TIA or stroke. RoPE score measured for this patient was 9 with an $88 \%$ chance of stroke being due to a PFO.

\section{Discussion}

Ischemic strokes are divided into multiple categories including large artery atherosclerosis, small vessel disease, and cardioembolic strokes which make up majority of the identifiable causes [7]. One of the most important factors for determining a stroke cause is the age of the patient. In younger population, specifically those less than 60 -yearsold, hematologic causes and structural causes are more common while arteriolosclerosis and atrial fibrillation are more common in in individuals older than 60-years-old [2]. Cryptogenic strokes are defined as any stroke that cannot be attributed to any specific cause despite adequate investigation. This can account for up to $30 \%$ of the strokes. Patient presenting to the hospital with symptoms of stroke must undergo investigation to determine the underlying cause due to the importance of time in stroke management. This involves performing an adequate history and physical examination and utilization of adequate imaging and laboratory modalities. Some of the standard evaluation tools used in stroke assessment include CT of the brain, MRI of the brain, vessel imagining using MRA or CTA, TTE, TEE, cardiac rhythms and complete blood count. If these modalities fail to establish the cause of stroke, then patients require further investigation such as a prolonged duration of cardiac rhythm monitoring and hematologic testing. When all these examinations fail to show a true cause for the stroke, then it is considered cryptogenic [2].

PFO is a connection between the left and right arteries that can allow blood or blood clots to travel paradoxically if the pressure in the right side is more than the left side of the heart. PFO is a common phenomenon and can be found in $25 \%$ of the general population [8]. Presence of $\mathrm{PFO}$ in patients who have cryptogenic strokes can range anywhere from $10-77 \%[9,10]$. The relationship becomes more striking when we look at patients under the age of 55 years who are 6 times more likely to have a PFO compared with patients who have a known cause of stroke. After the first episode of cryptogenic stroke, risk of having a repeat stroke increases on average $2 \%$ annually. Studies have shown that this number can be as high as $16 \%$ [11]. Hence, treatment and type of therapy plays an important role in these cases since patients who have an episode of stroke or TIA are much more likely to have a repeat episode of stroke which is even more true in those with PFO [12]. In 
addition, Risk of Paradoxical Embolus (RoPE) calculator is a useful tool that can be used to check for the likelihood that the stroke and the PFO are related. It considers age (younger age gives more points), infarct on imaging, smoking history, stroke or transient ischemic attack history, diabetes history, and hypertension with a maximum score of 10 . Due to the unique scoring feature, RoPE calculator can identify younger patients without conventional risk factors. The probability of finding a PFO increases from $23 \%$ (with scores 0 to 3 ) to $73 \%$ (with a score of 9 or 10 ), which correspond, respectively, to a PFO-attributable risk of $0 \%$ to $88 \%$ [11]. This makes RoPE calculator a useful tool when presented with a case of cryptogenic stroke.

Currently, majority of patients who have cryptogenic stroke and PFO are managed by antiplatelet and hyperlipidemia therapy. Until recently, in this category of patients, the consensus was not to close the PFO due to earlier studies not showing any benefit including CLOSURE I (Evaluation of the STARFlex Septal Closure System in Patients with a Stroke and/or Transient Ischemic Attack due to Presumed Paradoxical Embolism), PC (Percutaneous Closure of Patent Foramen Ovale in Cryptogenic Embolism), and RESPECT (Randomized Evaluation of Recurrent Stroke Comparing PFO Closure to Established Current Standard of Care Treatment). These trials failed to show superiority of closure over medical therapy [13-15]. Also, in a 2014 meta-analysis of randomized trials by Udell et al., it was concluded that at that time transcatheter closure of the PFO did not significantly reduce the short-term incidence of recurrent stroke or TIA [16]. However, more recently, there has been long-term follow up of patients who have undergone PFO closure and there have been multiple new studies that have investigated PFO closure outcomes. Mas et al. and Sondergaard et al. demonstrated that PFO closure reduced risk for ischemic stroke and new brain infarctions without significant increase in serious adverse events $[6,17]$. De Rosa et al. performed a systematic review and metaanalysis of PFO closure versus medical therapy in stroke patients with $\mathrm{PFO}$ and they concluded that compared to medical treatment, closure of PFO does prevent recurrent stroke and TIA [18].

The case reports presented in this paper are three prime examples of how a cryptogenic stroke on two different sides of the age spectrum can be managed. As was noted, in younger patients the likelihood that the cryptogenic stroke is secondary to PFO is much higher and when we look at the RoPE scores for these individuals. A RoPE score of 6 in case 1 suggests a $62 \%$ chance that the stroke is due to a PFO and an $8 \%$ risk of 2 year recurrence of stroke/TIA. A RoPE score of 9 in case 3 gave the patient an $88 \%$ chance of the stroke being due to a PFO. In case 2 , the RoPE score was 5 and a $34 \%$ chance that the stroke is due to a PFO and a $7 \%$ risk of 2 year recurrence of stroke/TIA. This is not surprising since the effect of age on the RoPE score is extremely high and younger individuals usually suffer from less chronic conditions, thus when they do present with a stroke/TIA and have a PFO present, the chances that the episode was secondary to the PFO is significantly higher. Naturally, with older patients, especially those greater than 60 -years-old, we should rule out secondary causes which might be absent when inpatient is critical, including atrial fibrillation or atrial flutter by utilizing a cardiac monitor. In younger patients, however, if the primary work up fails to show another cause for the stroke other than the PFO and cardiac monitor has failed to show arrythmias during the hospital course, it is much more likely that they would benefit from closure of PFO during the same hospitalization.

It has been already been established that in $50 \%$ of the cases, stroke in TIA patients occur within 48 hours of the TIA and up to $15 \%$ have a stroke within 3 months, thus prompt assessment is crucial [19].
This can be clearly seen in Case 1 where the patient did have a stroke approximately 36 hours after admission. When his imaging (which showed an old stroke) and his age are considered, it can be argued that the likelihood that the PFO was the culprit, is extremely high and the immediate closure of the PFO would benefit such patients. On the other hand, Case 2 was not as clear cut. Since patient was 66 years old and did not have many comorbid conditions, the possibility that there was an underlying secondary cause for the stroke was higher. Thus, he does require more extensive work up and assessment. He was discharged with cardiac event monitor to rule out atrial fibrillation or flutter episodes and will have the PFO closed if other causes are ruled out. In case 3 , the patient is young and has a very high RoPE score with lack of evidence for other causes, closure of the PFO does lead to a great outcome.

\section{Conclusion}

When considering PFO closure in stroke patients, especially those younger than 60 years old, who have a PFO, it is important to rule out large artery atherosclerosis, cardioembolic source, small vessel disease, dissection and hypercoagulable states as the causes for stroke. These should be worked up with the modalities previously mentioned. If they are negative, it is important to make sure hypertension and diabetes are well controlled and that there no other causes such as autoimmune diseases or drug/alcohol abuse. Atrial fibrillation/flutter must be ruled out and other contributing causes ruled out such as sepsis, endocarditis, valvular heart disease, liver disease or renal disorders. If these sources of stroke are ruled out, PFO closure should be performed. It should be also remembered that if factors such as previous thromboembolism, multifocal cerebral defects, a large PFO, or atrial septal aneurysm are present, it greatly favors early closure of PFO [20,21]. Thus, based on these observations, it can be deducted that a more prompt and decisive approach in PFO closure, especially in younger group of patients aged less than 60-years-old, especially those in the younger ages, can provide a much higher morbidity and mortality benefit. For future directions of the PFO closure and studies, it would be beneficial to see what the subset analysis shows based on different age groups of patients who have undergone PFO closure shows and study its long-term morbidity and mortality benefit.

\section{Disclosure}

All authors above take responsibility for all aspects of the reliability and freedom from bias of the data presented and their discussed interpretation and have no conflicts of interest or funding to disclose.

\section{References}

1. Taggart NWM, Reeder GS, Lennon RJ, Slusser JP, Freund MA, et al. (2016) Long-term follow-up after PFO device closure: Outcomes and complications in a single center experience. Catheter Cardio Inte 89: 124-133.

2. Saver J (2016) Cryptogenic stroke. NEJM 374: 2065-2074.

3. Blakenship RJ (2017) Patent foramen ovale closure to prevent secondary neurologic events. European J Intern Med 44: 1-11.

4. Steiner MM, Rundek T, Gan R, Chen X, Liguori C, et al. (1998) Patent foramen ovale size and embolic brain imaging findings among patients with ischemic stroke. Stroke 29: 944-948.

5. Saver JL, Carroll JD, Thaler DE, Smalling RW, MacDonald LA, et al. (2017) Long Term outcomes of patent foramen ovale closure or medical therapy after stroke. NEJM 377: 1022-1032.

6. Mas JL, Guillon GDB, Massardier E, Hosseini H, Mechtouff L, et al. (2017) Patent foramen ovale closure or anticoagulation vs. antiplatelets after stroke. NEJM 377: 1011-1021

7. Isabel C, Jean-Louis M (2016) Stroke prevention. Presse Med 45: 457-471. 
Citation: Pour-Ghaz I, Jackson C, Bhole R, Krishnan R, Pierce WF, et al. (2018) Cryptogenic Stroke and Significance of the Patent Foramen Ovale: A Case Series. J Clin Case Rep 8: 1167. doi: 10.4172/2165-7920.10001167

Page 4 of 4

8. Lim ST, Smith DR, Williams J, Navarro SG, McCabe J, et al. (2017) Clinical outcomes and a high prevalence of abnormalities on comprehensive arterial and venous thrombophilia screening in TIA or ischaemic stroke patients with a patent foramen ovale, an interatrial septal aneurysm or both. J Neuro Sci 377: 227-233.

9. Schulze V, Karathanos A, Brockmeyer M, Zeus T, Polzin A, et al. (2018) Patent foramen ovale closure or medical therapy for cryptogenic ischemic stroke: An updated meta-analysis of randomized controlled trials. Clin Res Cardiol 9: 745755.

10. Salem EA, Helmy T, Boakye EA, Alkhawam H, Lim M (2018) Patent foramen ovale closure versus medical therapy in cases with cryptogenic stroke, metaanalysis of randomized controlled trial. J Neurol 265: 578-585

11. Suradi HS, Hijazi ZM (2016) Patent foramen ovale stroke and device closure. Cardiol Clin 34: 231-240.

12. Dalen JE, Alpert JS (2015) Which patent foramen ovales need closure to prevent cryptogenic strokes? Ame J Med 131: 222-225.

13. Furlan AJ, Reisman M, Massaro J, Mauri L, Adams H, et al. (2012) Closure or medical therapy for cryptogenic stroke with patent foramen ovale. NEJM 366 991-999.

14. Meier B, Kalesan B, Mattle HP, Khattab AA, Hildick-Smith D, et al. (2013) Percutaneous closure of patent foramen ovale in cryptogenic embolism. NEJM 368: 1083-1091.
15. Carroll JD, Saver JL, Thaler DE, Smalling RW, Berry S, et al. (2013) Closure of patent foramen ovale versus medical therapy after cryptogenic stroke. NEJM 368: 1092-1100.

16. Udell JA, Opotowsky AR, Khairy P, Silversides CK, Gladstone DJ, et al. (2014) Patent foramen ovale closure vs. medical therapy for stroke prevention: Metaanalysis of randomized trials and review of heterogeneity in meta-analyses. Canadian J Cardiol 30: 1216-1224.

17. Søndergaard L, Kasner SE, Rhodes JF, Andersen G, Iversen HK, et al. (2017) Patent foramen ovale closure or antiplatelet therapy for cryptogenic stroke. NEJM 377: 1033-1042.

18. Rosa SD, Sievert H, Sabatino J, Polimeni A, Sorrentino S, et al. (2018) Percutaneous closure versus medical treatment in stroke patients with patent foramen ovale: A systematic review and meta-analysis. Ann Int Med 168: 343350 .

19. Cancelli I, Gigli GL, Perelli A, Zanchettin B, Canal G, et al. (2011) Incidence of transient ischemic attack and early stroke risk validation of the ABCD2 score in an Italian population-based study. Stroke 42: 2751-2757.

20. Mojadidi MK, Zaman MMO, Elgendy IY, Mahmoud AN, Patel NK, et al. (2018) Cryptogenic stroke and patent foramen ovale. JACC 71: 1035-1043.

21. Xin-Lin Z, Wang L, Xu B (2018) Percutaneous closure versus medical therapy for stroke with patent foramen ovale: A systematic review and meta-analysis. Cardiovasc Dis 18: 1-12. 